\title{
DIVERSIDAD DE INSECTOS DEL SUELO ASOCIADOS AL CULTIVO DE MAÍZ (Zea mays L.) EN LA MOLINA / LIMA / PERÚ
}

\section{DIVERSITY OF SOIL DWELLING INSECTS ASSOCIATED WITH CORN CROP (Zea mays L.) AT LA MOLINA / LIMA / PERU}

\author{
Carmen Livia ${ }^{1,2}$, Guillermo Sánchez ${ }^{1,3}$ y Luis Cruces ${ }^{1,4}$
}

\begin{abstract}
Resumen
Con el propósito de determinar la composición, abundancia y diversidad de insectos de suelo en un campo agrícola de producción de maíz (5 ha), en el distrito de La Molina (Lima, Perú), se instalaron 12 trampas de caída, las cuales fueron evaluadas semanalmente durante aproximadamente 4 meses (marzo-junio). Las muestras se procesaron separando los individuos por morfotipos, registrando la cantidad y la fecha de la colecta. Finalmente, se identificaron los ejemplares en el Laboratorio del Museo de Entomología Klaus Raven Büller con la ayuda de claves de identificación y, en algunos casos, comparación con los especímenes del museo. Se registraron individuos de los órdenes Orthoptera (Fam. Acrididae y Gryllidae); Dermaptera (Fam. Anisolabididae); Coleoptera (Fam. Carabidae, Staphylinidae, Tenebrionidae, Scarabaeidae y Elateridae); e Hymenoptera (Fam. Formicidae). El orden más abundante fue Hymenoptera con el género Linepithema sp. (Fam. Formicidae). El orden Coleoptera registró el mayor número de morfoespecies identificadas, entre las cuales Blennidus peruvianus fue el más predominante. El análisis de diversidad mostró un alto valor del índice de dominancia de Simpson $(0.91 \pm 0.006)$, mientras que el valor del índice de Shannon fue intermedio $(2.67 \pm 0.05)$. Los estimadores de Chao1 y ACE indicaron que la riqueza específica observada fue muy cercana a la estimada, obteniendo un esfuerzo de muestreo entre 81.9 y $84.7 \%$.
\end{abstract}

Palabras clave: diversidad, insectos de suelo, maíz, Perú, trampas de caída, Orthoptera, Dermaptera, Coleoptera, Hymenoptera.

\begin{abstract}
In order to determine the composition, abundance and diversity of soil dwelling insects in an agricultural field of corn production (5 ha), in the district of La Molina (Lima, Peru), 12 pit falls were installed, which were evaluated weekly for approximately 4 months (March-June). Collective samples were processed by separating individuals by morphotypes, recording the quantity and date of the collection. Finally, the specimens were identified in the Laboratory of the Klaus Raven Büller Entomology Museum with the help of identification keys and, in some cases, by comparison with museum specimens. Individuals of the orders Orthoptera (Families Acrididae and Gryllidae); Dermaptera (Family Anisolabididae); Coleoptera (Families Carabidae, Staphylinidae, Tenebrionidae, Scarabaeidae and Elateridae); and Hymenoptera (Family Formicidae) were identified. Order Hymenoptera was the most abundant with genus Linepithema sp. (Family Formicidae). Order Coleoptera registered the highest number of identified morphospecies, among which Blennidus peruvianus was the most predominant. The analysis of diversity gave a high value of Simpson's dominance index $(0.91 \pm 0.006)$, whereas the Shannon index value was intermediate $(2.67 \pm 0.05)$. The Chao1 and ACE estimators indicated that the specific richness observed in the field was very close to the estimated, giving a sampling effort between 81.9 and $84.7 \%$.

Key words: diversity, soil insects, corn, Peru, pit falls, Orthoptera, Dermaptera, Coleoptera, Hymenoptera.
\end{abstract}

\section{Introducción}

El maíz (Zea mayz L.) es uno de los cultivos anuales más sembrados en Perú con aproximadamente 570000 ha destinadas para maíz amarillo duro, choclo, amiláceo o chala (MINAGRI, 2018). Debido a su importancia, se han realizado muchos trabajos en este cultivo, entre estos, sobre la entomofauna asociada. Sin embargo, pocos son los estudios de insectos de suelo comparados con los que habitan la planta, y usualmente dichas investigaciones están enfocadas en grupos taxonómicos específicos (por ejemplo, Carabidae), debido al mayor número de individuos encontrados (Dicke \& Guthrie, 1988).

La agricultura es una de las actividades que generan mayor perturbación y pérdida en la artropofauna del suelo (Pyle et al., 1981). Por ello, las investigaciones 
referentes a la composición de la entomofauna epigea en los cultivos cumplen un rol importante en el manejo de los agroecosistemas debido a que brindan información relevante sobre las especies que habitan el suelo y con ello, inferir el papel que cumplen en la comunidad de insectos. Además, con los datos de abundancia y diversidad de las especies registradas, se pueden realizar estudios dirigidos a determinar el grado de alteración sufrido a través del tiempo por causa de las actividades humanas, utilizando aquellas especies que actúan como bioindicadores de calidad del suelo (Fernández-Herrera et al., 2011). Ello contribuirá a que se puedan tomar medidas más adecuadas para la conservación de la biodiversidad.

El empleo de trampas de caída ("Pitfall traps") es un método de bajo costo y fácil uso para investigar a los insectos epigeos, y es comúnmente utilizado en estudios de ecología (Siewers et al., 2014; Skvarla et al., 2014). Los insectos colectados con mayor frecuencia usando esta técnica pertenecen a las familias Carabidae, Staphylinidae, Elateridae y Scarabaeidae del orden Coleoptera (Backlund \& Marrone, 1997; Arbogast et al., 2000; Marinoni \& Ganho, 2003); la familia Formicidae del orden Hymenoptera (Fernández, 2003; Abensperg-Traun \& Steven, 2006); las familias Gryllidae y Acrididae del orden Orthoptera (Rohyani \& Ahyadi, 2017; Livia \& Sánchez, 2019) y las familias Anisolabididae y Labiduridae del orden Dermaptera (Schuller \& Sánchez, 2003). Estos conforman un grupo muy importante en los agroecosistemas debido a que son parte de la artropofauna que interviene en el procesamiento de recursos vegetales, ciclos de nutrientes y red trófica existente (Iannacone \& Alvariño, 2006).

El distrito de La Molina, ubicado en la provincia de Lima, Perú, es un área principalmente residencial (INEI, 2016). Sin embargo, posee una zona de producción de cultivos muy activa perteneciente a la Universidad Nacional Agraria La Molina, donde se producen diversos cultivos, siendo el maíz uno de los principales. Tal es así que durante todo el año se puede observar este cultivo en sus diferentes etapas fenológicas. Estos campos son conducidos de manera convencional, es decir, utilizando fertilizantes químicos, pesticidas, riego dirigido y uso de maquinaria agrícola (Sarmiento et al., 1970; Barraco \& Díaz-Zorita, 2005; Tejeda-Reyes et al., 2016), que, generalmente, tienen un efecto perjudicial sobre la entomofauna epigea (Pyle et al., 1981). Debido a ello, es necesario estudiar la diversidad de los insectos del suelo presente en los cultivos, lo que permitirá monitorear su grado de alteración a través del tiempo como consecuencia de las prácticas agrícolas empleadas. En este contexto, el objetivo del presente estudio fue determinar la composición, abundancia y diversidad de los insectos del suelo asociados al cultivo de maíz en el distrito de La Molina, Lima, Perú.

\section{Materiales y Métodos}

Localización

El presente estudio se realizó en un campo de maíz de 5 hectáreas, perteneciente al área agrícola de la Universidad Nacional Agraria La Molina (1204'55"S; 7656'53"O; 244 msnm) en Lima, Perú. Muestreo

El muestreo se llevó a cabo mediante el uso de trampas de caída, las cuales consistieron en envases trasparentes de polietileno $(10 \mathrm{~cm}$ de diámetro y $15 \mathrm{~cm}$ de alto), conteniendo $250 \mathrm{ml}$ de una solución preservante $(10 \%$ formol, agua y 5 gramos de detergente) (Leather, 2005). Estas trampas se quedaron en el campo durante toda la fenología del cultivo, desde una semana después de la siembra hasta una semana antes de la cosecha, periodo comprendido entre el 09 de marzo y 08 de junio de 2013. Al momento del ingreso de la maquinaria para la ejecución de las prácticas de cultivo y aporque, dichas trampas fueron retiradas $\mathrm{y}$ reinstaladas el mismo día.

El campo evaluado fue dividido en 6 sectores (S), considerando la pendiente y los bloques de irrigación (Figura 1). Se instalaron 12 trampas (dos por cada sector, colocadas sobre el "lomo del surco") uniformemente distribuidas y distanciadas unas de las otras por lo menos 75 metros, y alejadas de los márgenes del campo al menos 10 metros para evitar el 'efecto borde' (Leather, 2005).

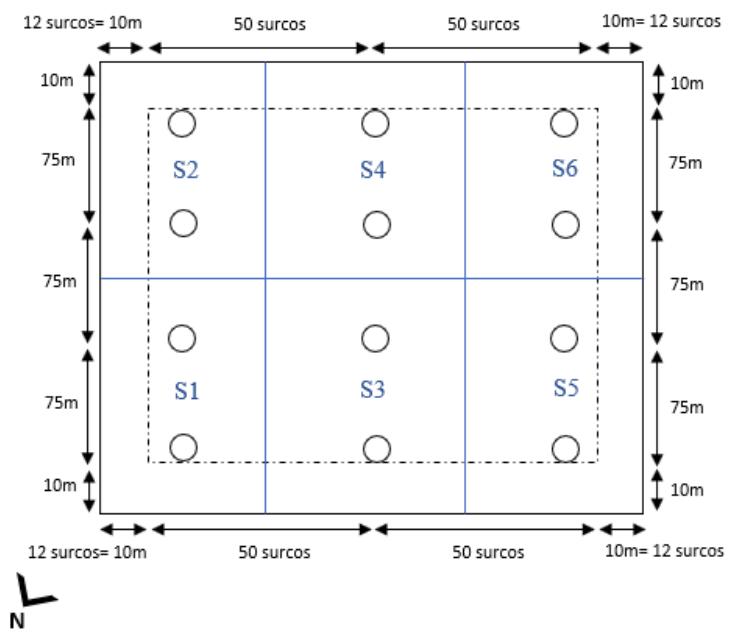

Figura 1. Distribución de las trampas de caída en el campo de maíz evaluado.

El contenido de las trampas fue periódicamente colectado (14 muestreos, un muestreo semanal) en envases herméticos de polietileno $(7 \mathrm{~cm}$ de diámetro y $5 \mathrm{~cm}$ de alto), cuidadosamente etiquetados con el número de trampa y la fecha de recolección. Luego, los envases correspondientes a las trampas de caída fueron lavados y llenados con una nueva solución preservante y reinstalados en el campo.

El cultivo fue conducido mediante prácticas agrícolas convencionales, es decir mediante el uso de 
fertilizantes químicos, pesticidas, riego por gravedad y uso de maquinaria agrícola.

Procesamiento de muestras

Las muestras colectadas en campo fueron procesadas en el Laboratorio de Investigación del Museo de Entomología "Klaus Raven Büller" del Departamento Académico de Entomología, Facultad de Agronomía, de la Universidad Nacional Agraria La Molina.

El contenido de las trampas de caída fue vertido a un tamiz de tela organza, y esta muestra fue cuidadosamente lavada con agua corriente para que los insectos colectados quedaran limpios y libres de los olores emanados por el formol. Estos insectos fueron colocados en otro envase hermético $(7 \mathrm{~cm}$ de diámetro y $5 \mathrm{~cm}$ de alto) limpio y con agua, para facilitar su posterior manipulación y evitar que se dañaran.

Utilizando estiletes y pinzas finas, los insectos colectados fueron trasladados a placas Petri para ser separados y agrupados de acuerdo a sus características morfológicas en morfotipos o morfoespecies (Oliver \& Beattie, 1993, 1996 a, b). Cada nueva morfoespecie fue fotografiada y codificada para facilitar la comparación con otros morfotipos similares. Asimismo, fueron contabilizados y registrados de acuerdo al grupo taxonómico que pertenecían y, finalmente, preservados en frascos herméticos de $10 \mathrm{ml}$ con etanol al $70 \% \mathrm{v} / \mathrm{v}$. Identificación

Los especímenes considerados en la presente investigación fueron aquellos insectos que se desarrollan y habitan principalmente en el suelo, los cuales fueron denominados en este estudio como "insectos de suelo".

Se utilizaron claves de identificación para determinar las familias, géneros y especies (cuando fue factible) de los siguientes órdenes: Coleoptera (Arnett et al., 2002; Martínez, 2005); Orthoptera (Naumann et al., 2000); Dermaptera (Naumann et al., 2000; Kočárek, 2014; Kočárek et al., 2015); Hymenoptera (Fernández, 2003; Fernández \& Sharkey, 2006). Algunas especies identificadas, en especial las del orden Coleoptera, fueron comparadas con los especímenes de la colección de referencia del Museo de Entomología Klaus Raven Büller.

Una muestra de 4 a 10 especímenes de cada especie identificada (según su abundancia en la colecta) fue montada y etiquetada siguiendo la técnica descrita por Triplehorn \& Johnson (2005), y depositada en la colección de referencia del Museo de Entomología Klaus Raven Büller.

Análisis de datos

La riqueza específica fue calculada mediante estimadores no paramétricos Chaol y Abundancebased Coverage (ACE), los cuales están basados en datos de abundancia de especies (Gotelli \& Colwell, 2011). Estos estimadores fueron obtenidos mediante el software "Estimate-S 9.1" (Colwell, 2013) para elaborar curvas de acumulación de especies y calcular los esfuerzos de muestreo, comparando la riqueza específica estimada con la riqueza de especies observada.

La diversidad alfa fue analizada mediante la estructura de la comunidad con curvas de rangos de abundancia, índice de Shannon para medir la equidad de especies e índice de dominancia de Simpson para evaluar si hay predominancia de ciertas especies en la comunidad. Estos fueron calculados mediante el Software R-Studio, versión 3.4.2 (R Core Team, 2017) (Paquetes: vegan, agricolae, biodiversity y MASS) (De Mendiburu, 2017; Oksanen, 2015; Kindt, 2018; Oksanen et al., 2018; Ripley et al., 2018).

\section{Resultados y discusión}

La campaña de evaluación del 09 de marzo al 08 de junio del 2013 en el cultivo de maíz en La Molina, dió como resultado la colección de 9132 especímenes que correspondieron a 34 morfoespecies de insectos de suelo pertenecientes a los órdenes Coleoptera, Dermaptera, Hymenoptera y Orthoptera (Tabla 1).

Riqueza específica

El orden Coleoptera tuvo mayor número de morfoespecies $(61.76 \%)$ con respecto a los órdenes Hymenoptera (29.41\%), Orthoptera (5.88\%) y Dermaptera (2.94\%) (Figura 2).

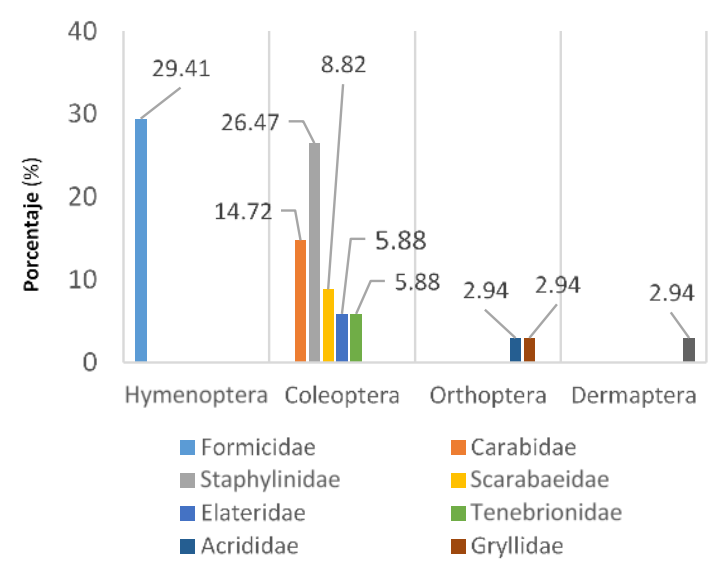

Figura 2. Distribución proporcional por categoría taxonómica del número de morfoespecies colectadas en el campo de maíz en La Molina, campaña de muestreo marzo-junio 2013.

De acuerdo a los estimadores de riqueza específica calculada mediante los índices Chao1 y ACE, el campo evaluado podría estar albergando alrededor de 40 especies de insectos de suelo, valor muy cercano al observado en el campo de maíz (Figura 3). Esto indica que los valores de esfuerzo de muestreo fueron de $84.7 \%$ para el estimador Chao1 y $81.9 \%$ para ACE. Abundancia relativa

Los rangos de abundancia proporcional de las 10 especies más abundantes son presentados en la Tabla 2, en la cual contrastan Linepithema sp. (Hymenoptera: Formicidae) y Blennidus peruvianus (Coleoptera: 
Carabidae) como las más abundantes, correspondiendo al $90.6 \%$ del total de individuos colectados.

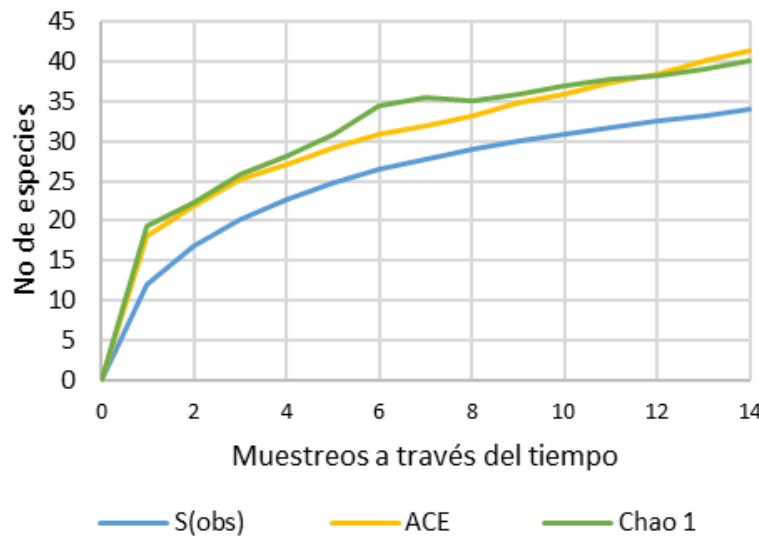

Figura 3. Curva de acumulación de especies observadas (obs) y esperadas (según los estimadores Chao1 y ACE) en el campo de maíz en La Molina, campaña marzo - junio 2013.

Tabla 2. Rangos de abundancia proporcional de las 10 especies más abundantes colectadas en el campo de maíz en La Molina, campaña de muestreo marzo - junio 2013.

\begin{tabular}{|c|c|c|c|}
\hline Morfoespecie & 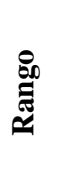 & 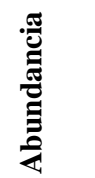 & 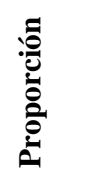 \\
\hline Linepithema sp. & 1 & 7046 & $77.2 \%$ \\
\hline Blennidus peruvianus & 2 & 1220 & $13.4 \%$ \\
\hline Aleocharinae Gen sp.1 & 3 & 112 & $1.2 \%$ \\
\hline Tetramorium sp. & 4 & 109 & $1.2 \%$ \\
\hline Blapstinus holosericius & 5 & 96 & $1.1 \%$ \\
\hline Gryllus sp. & 6 & 94 & $1.0 \%$ \\
\hline Tetragonoderus sp. & 7 & 78 & $0.9 \%$ \\
\hline Brachymyrmex sp. & 8 & 76 & $0.8 \%$ \\
\hline Epitragopsis sp. & 9 & 75 & $0.8 \%$ \\
\hline Formicinae Gen sp.1 & 10 & 56 & $0.6 \%$ \\
\hline
\end{tabular}

Al analizar la curva de rangos de abundancia (Figura 4), se observa una muy baja proporción de especies con una alta dominancia. Examinando la composición de la entomofauna colectada, se encontró que esta porción corresponde a tres morfoespecies (Linephitema sp., Blennidus peruvianus y Aleocharinae Gen. sp.1) las cuales en conjunto corresponden al $91.8 \%$ de la abundancia total de la comunidad colectada. La porción restante de la curva muestra un cambio en su pendiente, siendo esta mucho menos pronunciada, lo que corresponde a las especies más equitativamente distribuidas en la comunidad.

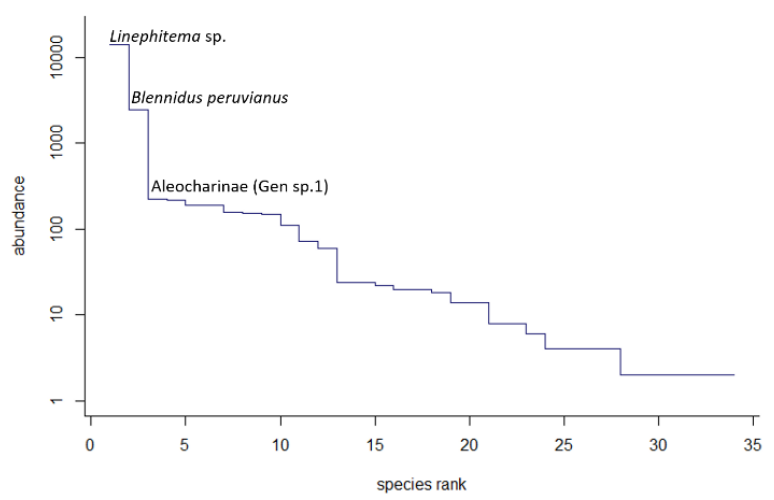

Figura 4. Curva de rango de abundancia de las morfoespecies encontradas en el campo de maíz en La Molina (distribución log-series).

Estructura de la comunidad

El valor obtenido del índice de Simpson fue de 0.91 \pm 0.006 , lo cual indica una alta dominancia de un pequeño grupo de especies en la comunidad.

El valor de índice de Shannon obtenido fue de 2.67 \pm 0.05 . Tomando en cuenta que los valores de este índice usualmente están entre 1.5 y 3.5 (Margalef, 1972), puede considerarse que la diversidad, en términos de equidad de especies, fue intermedia en el campo evaluado. Valores superiores a 4 son muy raros y solo ocurren cuando es muestreado un número muy elevado de especies (Margalef, 1972).

Variación específica

En el orden Hymenoptera se registraron 7323 individuos de la familia Formicidae, siendo Linepithema sp. la más abundante, correspondiendo al 96.2\% del total de hormigas contabilizadas (Tabla 1).

Es probable que la dominancia de Linepithema sp. sobre las otras morfoespecies de hormigas se deba a su lugar de anidación en la vegetación, viéndose menos perjudicadas cuando las actividades agrícolas perturbaban el suelo. Diferente fue el caso de los otros géneros encontrados, como Brachymyrmex sp. y Tetramorium sp. que anidan en el suelo o la vegetación, y Tranopelta sp. que lo hacen principalmente en el suelo. Además, especies del género Linepithema son dominantes debido a su capacidad de territorialidad, agilidad de desplazamiento en grandes grupos, dominancia de fuentes de alimento que los lleva a excluir a otras especies, y porque descienden fácilmente al suelo al percibir alimento (Fernández, 2003).

En cuanto a Coleoptera, se identificaron 1703 individuos de las familias Carabidae, Staphylinidae, Scarabaeidae, Elateridae y Tenebrionidae, de las cuales los miembros de Carabidae fueron los más abundantes correspondiendo al $76.45 \%$ del total de individuos contabilizados en este orden.

En la familia Carabidae se registraron 5 morfoespecies, de las cuales Blennidus peruvianus (Dejan) y Tetragonoderus sp. fueron las más abundantes, correspondiendo a $71.6 \%$ y $4.6 \%$ del total 
de carábidos colectados, respectivamente. Estos resultados concuerdan con otras investigaciones realizadas en La Molina, donde se registró $B$. peruvianus en los cultivos de maíz, camote, espárrago, frijol, tomate y lúcumo (Velapatiño, 1997; Livia \& Sánchez, 2019); y, aunque en baja proporción, a una especie del género Tetragonoderus en el cultivo de lúcumo (Livia \& Sánchez, 2019). Además, B. peruvianus fue registrada también con elevada abundancia en el cultivo de maíz en el Valle de Chancay, provincia de Huaral aledaño a la ciudad de Lima (Schuller \& Sánchez, 2003).

En la familia Staphylinidae se identificaron 9 morfoespecies, las cuales fueron determinadas solo hasta el nivel de subfamilia. Los morfotipos Alecharinae Gen. sp.1, Oxytelinae Gen. sp.1 y Staphylininae Gen. sp.1 fueron los más abundantes, abarcando el $56.8 \%, 18.2 \%$ y $15.2 \%$ del total de estafilínidos colectados, respectivamente. En Perú, se registran a Staphylininae y Aleocharinae como las subfamilias con el mayor número de especies reportadas, siendo Staphylininae la más predominante (Asenjo, 2004).

En la familia Tenebrionidae se registraron solo dos morfoespecies, identificadas como Epitragopsis sp. y Blapstinus holosericius (Laporte), abarcando el $43.9 \%$ y $56.1 \%$ del total de individuos colectados en este grupo taxonómico, respectivamente. Otro estudio realizado en el cultivo de lúcumo, también registró a estas especies en el distrito de La Molina (Livia \& Sánchez, 2019). La mayoría de los Tenebrionidae se caracterizan por ser de hábito alimenticio detritívoro (Crawford, 1991). Algunas investigaciones señalan que Epitragopsis y Blapstinus podrían alimentarse de residuos vegetales producidos por flores marchitas y polen liberado, así como también de materia orgánica (Rogers et al., 1978; Anteparra et al., 2013). En Perú, han sido reportados como plagas potenciales en el cultivo de camote (Rondón \& Vergara, 2004; Giraldo $\&$ Flores, 2016).

Con respecto a la familia Scarabaeidae, se identificaron a Anomala testaceipennis (Blanchard), Paranomala undulata (Melsheimer) y Ataenius sp. pero en baja incidencia en comparación con los morfotipos antes mencionados (9, 4 y 12 individuos, respectivamente). Especies de esta familia, tanto en estado larval como adulto, pueden alimentarse de estructuras vegetales subterráneas y aéreas (Mondaca, 2012), y pueden comportarse como plagas en diversos cultivos. En el maíz, $P$. undulata es registrada como plaga potencial (Valdivieso \& Núñez, 1984).

De la Familia Elateridae se colectaron solo a dos morfoespecies, identificadas como Conoderus sp. y Horistonotus sp. Su presencia en los muestreos no fue constante, registrándose solo a 7 y 1 individuos, respectivamente.

Del orden Orthoptera se colectaron 96 individuos, 97.9\% de estos identificados como Gryllus sp. de la familia Gryllidae, así como dos individuos de la familia Acrididae registrados como Gen. sp. Blanco \& Leyva (2009) reportaron a una especie de Gryllus en maíz. En investigaciones en otros cultivos como lúcumo y camote también se registró a Gryllus en el distrito de La Molina (Livia \& Sánchez, 2019; Livia-Tacza \& Sánchez, 2020). Los individuos de ambas familias son considerados fitófagos (Fontana et al., 2008; Pocco et al., 2010).

Finalmente, del orden Dermaptera solo se colectaron 10 individuos, identificados como Euborellia annulipes (Lucas, HF) de la familia Anisolabididae. El registro de esta especie es común en diversos estudios sobre entomofauna epígea (Schuller \& Sánchez, 2003; Rondón \& Vergara, 2004; Livia \& Sánchez, 2019; Livia-Tacza \& Sánchez, 2020). Esta especie tiene un hábito principalmente fitófago y de forma secundaria podría alimentarse de algún artrópodo del suelo (Rondón \& Vergara, 2004).

\section{Conclusiones}

En el cultivo de maíz estudiado en La Molina, Lima, Perú, se registraron insectos de suelo pertenecientes a los órdenes Orthoptera, Dermaptera, Coleoptera e Hymenoptera.

En el orden Coleoptera se registró el mayor número de morfoespecies, seguido por Hymenoptera, Orthoptera y Dermaptera. Los estimadores de Chao1 y ACE indicaron que la riqueza específica observada fue muy cercana a la estimada, obteniendo un esfuerzo de muestreo entre 81.9 y $84.7 \%$.

Los órdenes Hymenoptera y Coleoptera fueron los más abundantes, representados principalmente por Linepithema sp. (Fam. Formicidae) y Blennidus peruvianus (Fam. Carabidae), respectivamente.

El análisis de diversidad mostró un alto valor del índice de dominancia de Simpson, debido a la elevada abundancia en la comunidad de Linepithema sp., $B$. peruvianus y Aleocharinae Gen. sp.1; mientras que el valor del índice de Shannon fue intermedio.

\section{Literatura citada}

Abensperg-Traun M. \& Steven D. 2006. The effects of pitfall trap diameter on ant species richness (Hymenoptera: Formicidae) and species composition of the catch in a semi-arid eucalypt woodland. Austral Ecology, 20(2): 282-287. DOI: https://doi.org/10.1111/j.14429993.1995.tb00540.x.

Anteparra M., Redolfi I. \& Arellano C. 2013. Parepitragus pulverulentus and Epitragopsis olivaceus (Coleoptera: Tenebrionidae) in an ecological olive grove (Olea europaea L.) in the central coast of Peru. Revista Aporte Santiaguino, 6(1): 98-107. DOI: https://doi.org/10.32911/as.2013.v6.n1.522.

Arbogast R., Kendra P., Weaver D. \& Subramanyam B. 2000. Phenology and spatial pattern of Typhaea stercorea (Coleoptera: Mycetophagidae) infesting stored grain: estimation by pitfall trapping. Journal of Economic Entomology, 93(2): 240-251. DOI: https://doi.org/10.1603/0022-0493-93.2.240. 
Arnett R. H., Thomas M. C., Skelley P. E. \& Frank J. H. (eds) 2002. American beetles - Polyphaga: Scarabaeoidea through Curculionoidea. Washington - United States of America. $2 . \quad$ URL: https://www.zin.ru/animalia/coleoptera/addpages/Andre y_Ukrainsky_Library/References_files/Vandenberg02b. pdf.

Asenjo A. 2004. Lista preliminar de las especies de Staphylinidae (Coleoptera) registrada para Perú. Revista Peruana de Entomología, 44: 55-64. URL: http://www.revperuentomol.com.pe/index.php/rev-peruentomol/article/view/182/157.

Backlund D. \& Marrone G. 1997. New récords of the endangered american burying beetle, Nicrophorus americanus Olivier, (Coleoptera: Silphidae) in South Dakota. The Coleopterists Bulletin, 51(1): 53-58. DOI: https://www.jstor.org/stable/4009200.

Barraco M. \& Díaz-Zorita M. 2005. Nitrogen fertilization timing for corn crops in typic hapludolls. Ciencias del Suelo, 23(2): 197-203. URI: http://hdl.handle.net/10532/3794.

Blanco Y. \& Leyva Á. 2009. Las arvenses y su entomofauna asociada en el cultivo de maíz (Zea mays, L.) posterior al periodo crítico de competencia. Cultivos Tropicales, 30(1): 11-17. URI: http://repositorio.geotech.cu/jspui/handle/1234/2579.

Colwell R. 2013. EstimateS. Version 9.1.0. Department of Ecology and Evolutionary Biology, University of Connecticut, Storrs, USA CT, 06869-3043.

Dicke F.F. \& Guthrie W.D. 1988. The most important corn insects (Chapter 13). In: Sprague G.F. \& Dudley J.W. (Eds.) Corn and Corn Improvement. Vol. 18. 767-867. DOI: https://doi.org/10.2134/agronmonogr18.3ed.c13.

Fernández F. (ed.). 2003. Introducción a las Hormigas de la región Neotropical. Instituto de Investigación de Recursos Biológicos Alexander von Humboldt. Bogotá, Colombia. XXVI http://hdl.handle.net/20.500.11761/32961

Fernández F. \& Sharkey M.J. (eds). 2006. Introducción a los Hymenoptera de la Región Neotropical. Sociedad Colombiana de Entomología y Universidad Nacional de Colombia. Bogotá, Colombia. URI: http://hdl.handle.net/10261/79928.

Fernández-Herrera C., Combatt-Caballero E. \& RiveraJiménez H. 2011. Algunas características de la entomofauna de suelos sulfatados ácidos en Córdova, Colombia. Revista Mexicana de Ciencias Agrícolas, 2(3): 461-470.

URL: https://www.redalyc.org/articulo.oa?id=263119714012.

Fontana P., Buzzetti F. \& Mariño-Pérez R. 2008. Chapulines, Langostas, Grillos y Esperanzas de México. Guía fotográfica - Grasshoppers, Locusts, Crickets \& Katydids of Mexico. Photographic guide. URL: https://biodiversityassociation.org/wpcontent/uploads/2016/05/Grasshoppers-LocustsCrickets-Katydids-Of-Mexico.pdf

Giraldo A. \& Flores G. 2016. Peruvian Tenebrionidae: A review of present knowledge and biodiversity. Annales Zoologici, $\quad 66(4)$ : 499-513. DOI https://doi.org/10.3161/00034541ANZ2016.66.4.002.
Gotelli N. \& Colwell R. 2011. Estimating species richness (Chapter 4). In: Magurran A.E. \& McGill B.J. (Eds) Biological diversity: Frontiers in measurement and assessment. 39-54. URL: http://www.uvm.edu/ ngotelli/manuscriptpdfs/Chapter $\% 204$.pdf.

Iannacone J. \& Alvariño L. 2006. Diversidad de la artropofauna terrestre en la Reserva Nacional de Junín, Perú. Ecología Aplicada, 5(1,2): 171-174. DOI: http://dx.doi.org/10.21704/rea.v5i1-2.332.

INEI (Instituto Nacional de Estadística e Informática). 2016. Planos estratificados de Lima Metropolitana a nivel de manzana. Lima, Perú. URL: https://www.inei.gob.pe/media/MenuRecursivo/publicac iones_digitales/Est/Lib1403/libro.pdf.

Kindt R. 2018. Biodiversity R: package for community ecology and suitability analysis. R version, $2.10-1$. URL: https://CRAN.R-project.org/package=BiodiversityR.

Kočárek P., Dvorak L. \& Kirstova M. 2015. Euborellia annulipes (Dermaptera: Anisolabididae), a new alien earwig in Central European greenhouses: potential pest or beneficial inhabitant. Applied Entomology and Zoology, 50: 201-206. DOI: https://doi.org/10.1007/s13355-0150322-2.

Kočárek P. 2014. Earwigs (Dermaptera) of Socotra Island: checklist, distribution, and description of a new genus and four new species. Acta Entomologica Musei Nationalis Pragae, 54: 1-21. URL: https://www.biotaxa.org/AEMNP/article/view/9491.

Leather S. 2005. Insect sampling in forest ecosystems. Blackwell Science Ltd, United. DOI: 10.1002/9780470750513.

Livia C. \& Sánchez G. 2019. Soil insects associated with lucumo (Pouteria lucuma L.) trees in La Molina, Lima, Peru. Peruvian Journal of Agronomy, 3(1): 16-23. DOI: http://dx.doi.org/10.21704/pja.v3i1.1279.

Livia-Tacza C. \& Sánchez G. 2020. Soil arthropods associated with sweetpotato crop (Ipomoea batata L.) in La Molina, Lima, Peru. Peruvian Journal of Agronomy, 4(1): 1-9. DOI: http://dx.doi.org/10.21704/pja.v4i1.1438.

Margalef R. 1972. Homage to Evelyn Hutchinson, or why there is an upper limit to diversity. Connecticut Academy of Arts and Sciences. URI: http://hdl.handle.net/10261/166281

Crawford C.S. 1991. The community ecology of macroarthropod detritivores. In: Polis G.A. (ed.) The Ecology of Desert Communities. The University of Arizona Press. URL: https://www.cabdirect.org/cabdirect/abstract/199211587 74.

Marinoni R. \& Ganho N. 2003. Fauna de Coleoptera no Parque Estadual de Vila Velha, Ponta Grossa, Paraná, Brasil. Abundância e riqueza das famílias capturadas através de armadilhas de solo. Revista Brasileira de Zoologia, 20(4): 737-744. DOI: https://doi.org/10.1590/S0101-81752003000400029.

Martínez C. 2005. Introducción a los escarabajos Carabidae (Coleoptera) de Colombia. Instituto de Investigación de Recursos Biológicos Alexander von Humboldt. Bogotá D. C., Colombia. URI: http://hdl.handle.net/20.500.11761/9488. 
Naumann I.D., Came P.B., Lawrence J.F., Nielsen E.S., Spradbery J.P., Taylor R.W., Whitten M.I. \& Littlejohn M.J. (Eds). 2000. The Insects of Australia. Melbourne University Press. Volume 2.

De Mendiburu F. 2017. Agricolae: Statistical procedures for agricultural research. R package version, 1.2(8).

MINAGRI (Ministerio de Agricultura y Riego). 2018. Encuesta nacional de intenciones de siembra 2018. Sistema Integrado de Estadísticas Agrarias. Lima, Perú. URL:

http://siea.minagri.gob.pe/siea/sites/default/files/Lineami entos\%20Metodologicos\%20ENIS\%202018.pdf.

Mondaca J. 2012. Paranomala undulata peruviana (Scarabaeidae: Rutelinae): Un nuevo escarabajo exótico introducido en Chile. Revista Chilena de Entomología, 37: 75-80. URL: https://www.biotaxa.org/rce/article/view/29208.

Oksanen J. 2015. Multivariate analysis of ecological communities in R: vegan tutorial. R package [R]. URL: https://www.mooreecology.com/uploads/2/4/2/1/242139 70/vegantutor.pdf.

Oksanen J., Blanchet F., Michael F., Kindt R., Legendre P., Dan McGlinn P., Minchin P.R., O'Hara R.B., Simpson G.L., Solymos P., Stevens M.H.H., Szoecs E. \& Wagner H.. 2018. Package 'vegan'. Community ecology package, version 2.5(3). URL: https://CRAN.Rproject.org/package=vegan.

Oliver I. \& Beattie A.J. 1993. A Possible Method for the Rapid Assessment of Biodiversity. Conservation Biology, 7: 562-568. DOI: https://doi.org/10.1046/j.1523-1739.1993.07030562.x.

Oliver I. \& Beattie A.J. 1996a. Designing a cost-effective invertebrate survey: a test of methods for rapid assessment of biodiversity. Ecological Applications, 6: 594-607. DOI: https://doi.org/10.2307/2269394.

Oliver I. \& Beattie A.J. 1996b. Invertebrate morphospecies as surrogates for species: a case study. Conservation Biology, 10: 99-109. DOI: https://doi.org/10.1046/j.1523-1739.1996.10010099.x.

Pocco M., Damborsky M. \& Cigliano M. 2010. Comunidades de ortópteros (Insecta, Orthoptera) en pastizales del Chaco Oriental Húmedo, Argentina. Animal Biodiversity and Conservation, 33(2): 119-129. URL: http://abc.museucienciesjournals.cat/files/ABC-33-2-pp119-129.pdf.

Pyle R., Bentzien M. \& Opler P. 1981. Insect Conservation. Annual Review of Entomology. 26: 233-258. DOI: https://doi.org/10.1146/annurev.en.26.010181.001313.

R Core Team. 2017. R: a language and environment for statistical computing. R Foundation for Statistical Computing, Vienna Available in http://www.Rproject.org/.

Ripley B., Venables B., Bates D., Hornik K., Gebhardt F. \& Ripley M. 2018. Package 'mass'. CRAN Repos. Httpcran R-Proj. project.org/web/packages/MASS/MASS.pdf. https://cran.r-

Rogers L., Woodley N, Sheldon J. \& Uresk V. 1978. Darkling Beetle Populations (Tenebrionidae) of the Hanford Site in Southcentral Washington. Pacific Northwest Laboratories, Richland, Washington, USA. URL: https://www.osti.gov/servlets/purl/5041122.
Rohyani I.S. \& Ahyadi H. 2017. Short Communication: Diversity and abundance of soil insects at Jeruk Manis Protected Forest in East Lombok (Indonesia) using several trapping methods. Biodiversitas, 18(2): 809-812. DOI: https://doi.org/10.13057/biodiv/d180253.

Rondón S. \& Vergara C. 2004. Diversidad de artrópodos del suelo en cuatro cultivares de camote Ipomoea batatas (L.) Lam. (Convolvulaceae) en el valle de Cañete, Lima, Perú. Revista Peruana de Entomología, 44: 73-80. URL: https://sisbib.unmsm.edu.pe/BVRevistas/entomologia/v4 4/pdf/a12v44.pdf.

Sarmiento J., Cisneros F., Millones I. \& La Rosa J. 1970. Ensayos de campo para el control químico de Spodoptera frugiperda (J.E. Smith) y su efecto sobre Diatraea saccharalis F. en maíz. Revista Peruana de Entomología, 13(1): 64-69. URL: https://www.revperuentomol.com.pe/index.php/revperu-entomol/article/view/469.

Schuller S. \& Sánchez G. 2003. Los artrópodos de suelo depredadores en agroecosistemas de maíz en el Valle de Chancay, Lima, Perú. Revista Peruana de Entomología, 43: 47-57. URL: https://sisbib.unmsm.edu.pe/BVRevistas/entomologia/v4 3/pdf/a07v43.pdf.

Siewers J., Schirmel J. \& Buchholz S. 2014. The efficiency of pitfall traps as a method of sampling epigeal arthropods in litter rich forest habitats. European Journal of Entomology, 111(1): 69-74. DOI:10.14411/EJE.2014.008.

Skvarla M., Larson J. \& Dowling A. 2014. Pitfalls and preservatives: A Review. Journal of the Entomological Society of Ontario, 145: 15-43. URL: https://www.entsocont.ca/uploads/3/0/2/6/30266933/skv arla_2014_final.pdf.

Tejeda-Reyes M., Solis-Aguilar J., Díaz-Nájera J., PeláezArroyo A., Ayvar-Serna S. \& Mena-Baheba A. 2016. Evaluación de insecticidas en el control de gusano cogollero Spodoptera frugiperda J. E. Smith (lepidoptera: Noctuidae) en maíz en Cocula, Guerrero. Entomología Mexicana, 3: 391-394. URL: http://www.socmexent.org/entomologia/revista/2016/EA /Em\%20391-395.pdf.

Triplehorn C.A. \& Johnson N.F. 2005. Borror and DeLong's Introduction to the Study of Insects. Seventh Edition. Thompson Brooks/Cole (U.S.A.). URL: https://www.cengage.co.uk/books/9780030968358/.

Valdivieso L. \& Núñez E. 1984. Plagas del maíz y sus enemigos naturales. Manual técnico $\mathrm{N}^{\circ}$ 4. IICA. Lima, Perú. URI: https://repositorio.iica.int/handle/11324/8762.

Velapatiño J. 1997. Algunos artrópodos presentes en el suelo del área agrícola de la Universidad Nacional Agraria La Molina. Revista Peruana de Entomología, 40: 80-90. URL:

https://sisbib.unmsm.edu.pe/BVRevistas/entomologia/v4 0/pdf/a25v40.pdf. 
INSECTOS DEL SUELO ASOCIADOS AL CULTIVO DE Zea mays EN LIMA - PERÚ Agosto - Diciembre 2020

Tabla 1. Morfoespecies colectadas en el cultivo de maíz en La Molina, campaña marzo - junio del 2013.

\begin{tabular}{|c|c|c|c|}
\hline Orden & Familia & Morfoespecie & No de individuos \\
\hline \multirow{2}{*}{ Orthoptera } & Acrididae & 1.Gen. sp. & 2 \\
\hline & Gryllidae & 2.Gryllus sp. & 94 \\
\hline Dermaptera & Anisolabididae & 3.Euborellia annulipes & 10 \\
\hline \multirow{21}{*}{ Coleoptera } & \multirow{5}{*}{ Carabidae } & 4.Tetragonoderus sp. & 78 \\
\hline & & 5.Blennidus peruvianus & 1220 \\
\hline & & 6.Tetracha chilensis & 2 \\
\hline & & 7.Gen. sp. & 1 \\
\hline & & 8.Notiobia peruviana & 1 \\
\hline & \multirow{9}{*}{ Staphylinidae } & 9.Aleocharinae Gen. sp.1 & 112 \\
\hline & & 10.Aleocharinae Gen. sp.2 & 3 \\
\hline & & 11.Aleocharinae Gen. sp.3 & 1 \\
\hline & & 12.Aleocharinae Gen. sp.4 & 2 \\
\hline & & 13.Aleocharinae Gen. sp.5 & 4 \\
\hline & & 14.Aleocharinae Gen. sp.6 & 7 \\
\hline & & 15.Oxytelinae Gen. sp.1 & 36 \\
\hline & & 16.Staphylininae Gen. sp.1 & 30 \\
\hline & & 17.Paederinae Gen. sp.1 & 2 \\
\hline & \multirow{3}{*}{ Scarabaeidae } & 18.Ataenius sp. & 9 \\
\hline & & 19.Anomala testaceipennis & 4 \\
\hline & & 20.Paranomala undulata & 12 \\
\hline & \multirow{2}{*}{ Elateridae } & 21.Conoderus sp. & 7 \\
\hline & & 22.Horistonotus sp. & 1 \\
\hline & \multirow{2}{*}{ Tenebrionidae } & 23.Epitragopsis sp. & 75 \\
\hline & & 24.Blapstinus holosericius & 96 \\
\hline \multirow{11}{*}{ Hymenoptera } & \multirow{11}{*}{ Formicidae } & 25.Tetramorium sp. & 109 \\
\hline & & 26.Brachymyrmex sp. & 76 \\
\hline & & 27.Linepithema sp. & 7046 \\
\hline & & 28.Tranopelta sp. & 10 \\
\hline & & 29.Formicinae Gen. sp.1 & 56 \\
\hline & & 30.Formicinae Gen. sp.2 & 12 \\
\hline & & 31.Formicinae Gen. sp.3 & 1 \\
\hline & & 32.Formicinae Gen. sp.4 & 11 \\
\hline & & 33.Dolichoderinae Gen. sp.1 & 1 \\
\hline & & 34.Gen. sp. & 1 \\
\hline & & Total & 9132 \\
\hline
\end{tabular}

\footnotetext{
${ }^{1}$ Docente de la Universidad Nacional Agraria La Molina, Av. La Molina s/n. Lima - Perú.

2 clivia@lamolina.edu.pe.

${ }^{3}$ guillesanchezv@lamolina.edu.pe.

${ }^{4}$ luiscruces@lamolina.edu.pe.
} 